\title{
WHEN AND WHY DO CONTROLLERS MARK FLIGHT PROGRESS STRIPS? A LOOK AT LIVE TRAFFIC
}

\author{
Francis T. Durso, Peter J. Batsakes, Jerry M. Crutchfield \& Justin Braden \\ University of Oklahoma \\ \& \\ Carol A. Manning \\ Federal Aviation Administration Civil Aeromedical Institute
}

\begin{abstract}
Subject matter experts were employed to record observations of flight progress strip marking across 5 en route ATC facilities. Approximately 220 hours of ATC observations were recorded establishing a group of high frequency/high importance markings. These markings were perceived by controllers to provide performance benefits through externalizing memory and communication. The results are discussed in relation to possible electronic alternatives to flight progress strips.
\end{abstract}

Air Traffic Control (ATC) in the United States is accomplished by highly trained men and women using sophisticated technologies and intricate procedures to accomplish the safe and expeditious movement of the nation's air traffic. Due to projected increases in traffic volume, the FAA is in the process of modernizing the technologies used by controllers. The overarching goal is to increase the overall efficiency and expedite the movement of traffic through the Nation's airspace.

With this modernization effort comes an increased reliance upon automated alternatives to previously manual tasks. Often, little is known about the full impact of automation on an operator's performance until after its introduction. While automation may free the operator of unimportant or repetitive task components, it may also reduce an operator's situation awareness and compromise system safety (Hopkin, 1995). The current research was conducted in order to assess flight progress strip (FPS) marking among en route air traffic controllers in an effort to guide in the design process of an automated electronic alternative.

Currently, en route controllers have access to computer-augmented radar information displayed over a Situational Display and can use data entry and display devices to enter and retrieve information about an aircraft's flight plan. In addition, controllers have access to small pieces of paper (FPSs) that contain pertinent information about the pilot's flight plan. The controller is required to manage and update the strips in order to reflect pilot requests, coordinations and control clearances.

Strip marking requirements exist partly to satisfy the need to maintain a legal record of control activities should an operational error occur. Because most flight information regarding pilot-controller interaction is stored electronically, strip marking no longer serves the legal record function that it once did. However, most en route facilities still require the management and marking of strips during control operations, often a time consuming and distracting activity. Thus, designers of new ATC technologies have suggested the replacement of FPSs with a flight information system that displays updated information automatically or through keyboard input.

It is not clear, however, that such a replacement would be entirely beneficial. Luff, Heath and Greatbatch (1992) delineated five disadvantages of electronic presentation to paper: 1) keyboard entries are more difficult, 2) the range of entries is restricted, 3) computer entries restrict the sequence of input, 4) offer fewer ways of differentiating a document, and 5) restrict the mobility of information through the workplace. Furthermore, Hutchins (1995) suggested that technological aids can come to serve a cognitive function in ways other than originally intended. It has been suggested that FPS marking may have evolved in a way that benefits the controllers through a reduction of cognitive demands (Vortac, Edwards, Fuller and Manning,1993). However, empirical studies considering the ancillary cognitive benefits of FPS marking (Albright, Truitt, Barile, Vortac \& Manning, 1995; Vortac, Barile, Albright, Truitt, Manning \& Bain, 1996; Vortac et al., 1993) have shown no clear benefits to control performance in strip marking vs. non-strip marking conditions. It should be noted, however, that the previous studies investigating the possible benefits of FPS marking were conducted in simulated environments over short periods of time and may not have demonstrated realistic situations in which strip marking may be essential. Thus, it was a goal of the present study to sample a broad range of FPS marking activities during the observation of live ATC activities. Trained subject matter experts (SMEs) were used in order to collect the observational data. In addition to the observations of live strip markings, the perceived cognitive benefits and importance of strip activities were obtained through interviewing the controllers who marked the strips.

\section{METHOD: OBSERVATIONS}

\section{Facilities and Expert Observers}

Observations occurred across five different ARTCC facilities: Kansas City (ZKC), Chicago (ZAU), Atlanta (ZTL), Washington D.C. (ZDC) and Cleveland (ZOB) centers. Observations occurred over 3 consecutive weekdays (morning through early evening shifts).

Eight Certified Professional Controllers (CPC, $\underline{M}=$ 18.25 years, $\underline{\mathrm{SD}}=8.48$ years) were recruited from $\mathrm{ZKC}$ and ZAU en route facilities. Observer teams consisted of equal numbers of area supervisors as well as National Air Traffic Controllers Association (NATCA) union representatives. Schedules were arranged such that none of the SMEs observed control activities at their own home facilities. Prior to data 
collection, each of the observers participated in flight strip observational training at the Radar Training Facility (RTF) at the FAA's Mike Monroney Aeronautical Center in Oklahoma City. Training occurred over a 2-day period and consisted of having the group observe and record strip markings during 10minute simulations of ATC operations. Results of the training observations were compared among the expert observers until a consistent set of observational data collection protocols was established.

\section{Observational Materials}

During each observational session at a facility (12 observations per session), the trained observers were provided with a booklet containing strip marking observation forms. Each was used to record all strip markings that occurred during a 10-minute period within a particular sector. The form was constructed initially by listing markings documented in national and facility strip marking guides and from input provided by en route instructors from the FAA Academy in Oklahoma City. The forms were subsequently modified after consultation with the expert observers both before and after the observational training sessions. Each form was constructed to contain 10 main areas of information: 1) an information area that provided the observer with the area of specialization to be observed, as well as the sector number, position (R-side, D-side, tracker) and staffing (1, 2, 3, 4+ controllers), 2) an Issued Clearance markings area (Route, Heading, Altitude, Speed, Approach/Departure, Holding), 3) a Coordinated Clearance markings area, 4) a Planned Clearances Marking area, 5) an Incoming/Outgoing Radar Communications marking area (Incoming Radar/Communications, Outgoing Radar, Outgoing Communications), 6) a Non-Clearance Coordinations area (Control Released/Received, Pointout), 7) an Information Updates area (Times/Time Updates, Eliminations/Revisions of Control Information, Check Altitude When Level/Leaving), 8) an "other" strip markings area, 9) a Non-Marking StripRelated Activities area (offsets, moves, points) and 10) a relevant environmental factors area.

\section{Procedure}

Observations at each center occurred over a 3-day period. Each observer collected data during 2 sessions per day. Thus, 24 sessions or 2880 minutes ( $48 \mathrm{hrs}$ ) of strip marking observations were recorded at each facility. Only strip markings observed at sectors within a single area of specialization were recorded by a particular observer during an observational session. Observers proceeded to randomly determined sectors within the area of specialization and observed the actions and markings at randomly chosen positions. As per previous agreement with NATCA, the observers did not listen to the radio and communications information (i.e., the observer did not "plug in"). Initially, the observers recorded the position observed (i.e., R-side, D-side, other), the start time of the observational period, the range used by the R-side controller, and the number of Full Data Blocks (FDBs) appearing on the radar scope at the beginning of the observation. Until the end of the observation period, the observer recorded each time the controller marked a strip or performed some other strip-related activity by marking a tally in the appropriate space provided on the observation form. At the end of the observational period, the observers marked the stop time as well as any of the environmental factors that may have applied.

\section{RESULTS: OBSERVATIONS}

There were 13,200 minutes of observation included in the analysis. Observations were excluded if we were unable to determine the position observed from the data or if training occurred at the sector during the time of observation. Sectors were staffed by individuals $66.7 \%$ of the time, and by teams, $33.3 \%$ of the time. Observations of high altitude $(52.9 \%)$ and low altitude (47.1\%) sectors were approximately equal.

The strip markings/activities recorded by our observers were initially rank-ordered according to frequency of observation. A mean-split was then conducted on the data in order to separate higher frequency from lower frequency strip markings or activities. Table 1 contains the strip markings/activities in order of frequency of observation. Overall, a large number of Incoming/Outgoing Radar/Communication markings (2.79 marks per 10 minute period) were observed, more than any other strip marking or activity. This was followed by non-strip marking activities (2.58), Issued Clearances (0.79), Information Updates (0.67) and Non-clearance Coordinations (0.28).

Table 1. Frequencies of strip markings/activities strip marking/activity observed frequency

Incoming/Outgoing Radar

Communications

Non-marking Strip Related

Activities

Issued Clearances

Information Updates

Non-Clearance Coordinations

Coordinated Clearances

Planned Clearances

Although frequencies of observed strip markings and activities provide an important accounting of strip marking activities across the five ARTCC facilities, they do not necessarily provide information regarding the perceived importance of those strip markings to actual ATC operations. For instance, it is not clear whether controllers make these 
marks because they are important to their control performance or merely to satisfy facility strip marking requirements. In order to account for perceived importance of strip markings, 10 Certified Professional Controllers from various ARTCC facilities were asked to rank-order strip markings/activities on a scale of 1 (low importance) to 100 (high importance).

A mean-split was also performed on the geometric means of the importance ratings. Through combining frequency with the importance rating data, we defined a group of strip markings/activities that emerged as being both high frequency and of high importance. These markings/activities were primarily comprised of Issued Clearance markings (i.e., Altitude, Route, etc.). However, there were also some Coordination markings as well as Information Updates represented among the highest frequency/most important strip markings/activities. As can be seen from Table 1, the highest frequency markings/activities (Incoming

Radar/Communications \& Non-Marking Strip Related Activities) were not considered important by our SME raters.

\section{METHODS: INTERVIEWS}

\section{Interviews Procedure (ZKC, ZAU, ZTL)}

The observers at ZKC, ZAU and ZTL were instructed to select two FPSs per observational session as subject matter for follow-up interviews. FPSs were selected based on representativeness of sector traffic during observation as well as uniqueness of markings. The goal of the selection procedure was to obtain a record of controller perceptions for as wide a range of different strip markings/activities as possible. After selecting the subject matter for an ensuing interview, the observer arranged to have the FPS of interest retained and delivered to the interview area and asked the observed controller to participate in the interview procedure during an upcoming break period. Participation in interviews was optional.

The first interview procedure was used in order to explore the range of benefits provided by strip markings/activities and to see what benefits would naturally emerge. We used an open-ended interview procedure. The interview questions were developed in accordance with established cognitive interviewing techniques (Geiselman \& Fisher, 1997; Klein, Calderwood \& MacGregor, 1989) that have been demonstrated to provide more complete and accurate details than other interviewing procedures.

The first step of the interview procedure was to have the controller indicate those markings that he or she made (as opposed to a teammate or relieved controller) by highlighting them on a photocopy of the target strip. The controller was then asked to provide the chronological order in which he or she made the strip markings as well as provide an indication of whether he or she resequenced, offset or pointed to the strip. We attempted to recover the original context by asking controllers to construct a timeline that included the occurrence of each strip marking/activity as well as the events leading up to each occurrence. The timelines, themselves, were not subjected to analysis.
From this timeline, a single target strip marking/activity was then selected for further analysis. The controllers initially indicated whether the target strip marking was made solely because it was required by facility or national strip marking order or if it provided some cognitive benefit to control performance. Controllers indicating a benefit beyond satisfying strip marking requirements then wrote responses to open-ended questions designed to elicit the perceived cognitive benefits of the target strip marking/activity.

\section{RESULTS}

Of the 196 Controllers invited to participate in the interviews procedure, 186 complied. The answers from the questionnaire data from the preliminary interviews procedure were initially parsed into idea units. Each of these idea units was then used to code answers to the responses along a perceived benefits dimension. Two members of the research team coded the responses. Initial agreement between researchers reached $87.5 \%$. Differences between coders were then negotiated until full agreement was reached.

For the $84 \%$ of controllers who indicated some benefit of the target marking/activity, the answers to the question were further coded according to categories that emerged from the responses. These included: 1) the target strip marking/activity facilitated communication between teams of controllers or between the controller making the target strip marking/activity and the relieving controller, 2) the target strip marking helped to save time or eliminate unnecessary repetitive actions among individuals or control teams, 3) the target strip marking/activity provided an external memory aid or an external reference to important sources of information, and 4) the target strip marking/activity enabled the controller to organize information or aided in locating the strip. Answers to the perceived benefits question could be coded as fitting more than one category of perceived benefits if the answer reflected multiple benefits. Figure 1 shows the perceived benefits for the highest frequency/most important markings described earlier.

\section{Interviews Procedure (ZDC, ZOB)}

The second interviews procedure (ZDC, $\mathrm{ZOB}$ ) was conducted in order to capture and quantify the specific benefits of each mark/activity. We used a closed-ended Likert-type format based on the analysis of the initial interviews. Interviews at $\mathrm{ZDC}$ and $\mathrm{ZOB}$ allowed us to quantify more precisely the benefits that emerged and to determine the extent to which the benefits were perceived in individually staffed and team staffed situations. Of the 190 controllers invited for interviews, 109 complied. During the interview, the controller indicated which markings he or she made and indicated the chronological order of the markings as with the first interview procedure. Two markings (target markings A and B) were then selected as the subject matter for the ensuing interview. 


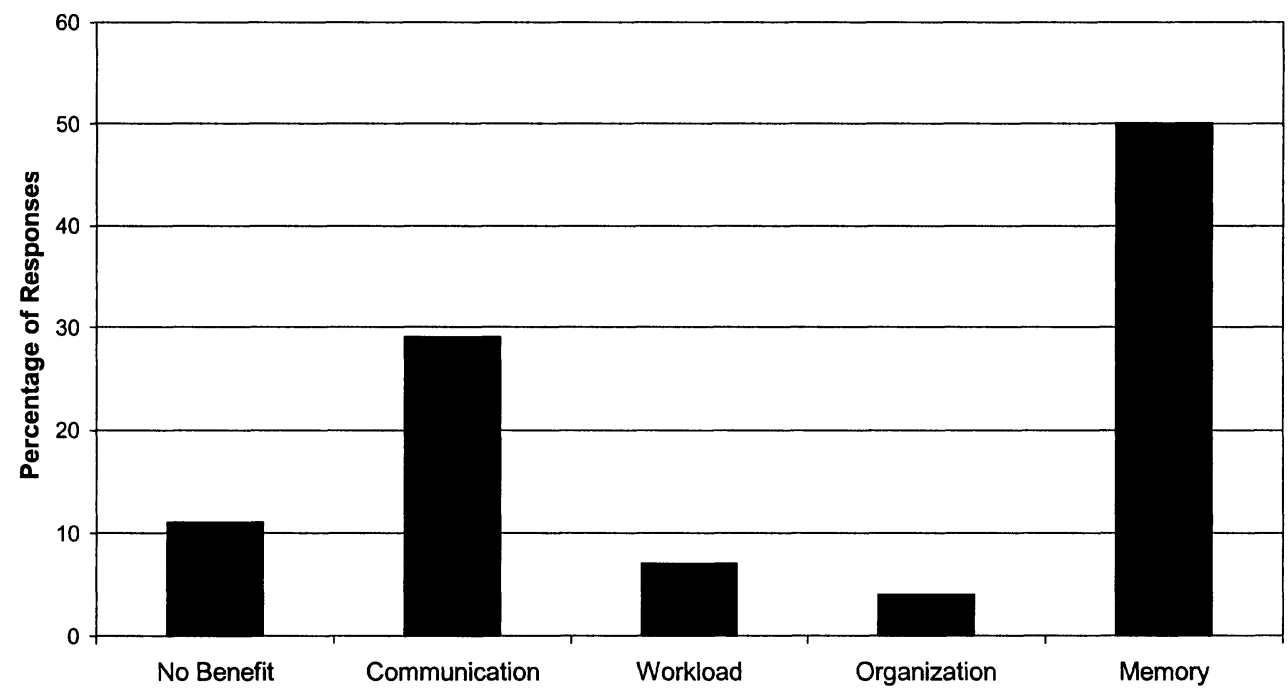

Figure 1. Benefits of most frequent and important markings/activities

The questionnaire for the second interview procedure included 8 probes that presented interviewees with prepared statements, 2 probes for each of the benefit categories elicited from the first set of interviews: Memory (e.g., The target marking/activity was beneficial because it allowed me to refer to information I would have otherwise had to remember), Communication (e.g., ... was beneficial because it allowed me to communicate information with sector teammates or other controllers without directly speaking with them), Workload (e.g., . . . I saved excess work for myself or my sector teammate), and Organization (e.g., . . . helped me organize control-related information in a more meaningful way). The interviewees were to reflect their level of agreement with the statement by circling any number between 1 "Strongly disagree" and 7, "Strongly agree", with 4 reflecting "No opinion."

\section{RESULTS}

Overall, there were 210 target marks resulting from the follow-up interviews (ZDC and ZOB). Of those marks, 155 were perceived by the interviewee as beneficial and were subsequently the subject of the remaining interview probes.

A combined score was calculated for each of the 4 benefits categories by averaging the results of the two answers within each benefit category. The results of the Likert questionnaires for the 7 highest frequency/most important marks are presented in Figure 2. As can be seen from the figure, communications and memory were the greatest benefits offered by the 7 most frequent and most important markings. However, those interviewed also saw benefits in workload offset and the organization of information. Thus, the second set of interview data are in general agreement with the results of the initial, exploratory interviews dataset.
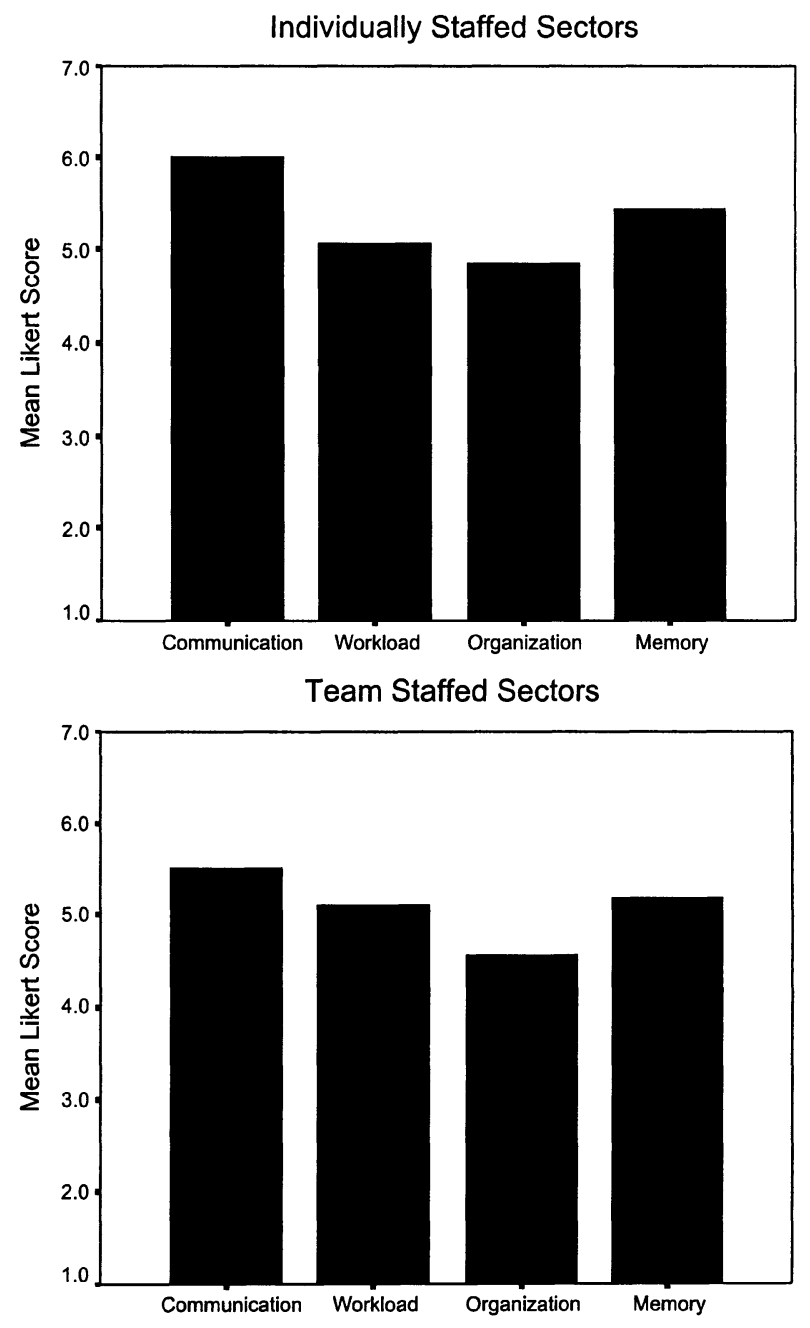

Figure 2. Likert responses for individuals and teams 


\section{CONCLUSIONS}

The present study represents the first major investigation of strip markings during the control of live traffic. While previous studies investigating the role of strip marking during simulated exercises have suggested that strip marking is not an irreplaceable component of control activities, the current study suggests that the benefits offered by some strip markings need to be accounted for within an automated information system.

The present investigation uncovered a group of high frequency/high importance strip markings used by en route ATC while controlling live traffic. Those markings mostly involved the indication that a control clearance had been issued (Altitude, heading, route, speed). Other frequent and important markings were associated with an indication that coordinations had or needed to be accomplished within the ATC system (altitude coordinations, Pointouts) while another important and frequent marking simply reflected that established control information had changed (eliminations/revisions of control information). Subsequent interviews with en route controllers revealed these marks to be especially beneficial in terms of their communication value as well as their ability to represent information externally so that it would not be necessary for controllers to remember important information during busy control operations. While these were the main benefits offered by these markings, controllers also suggested that the markings offset workload and helped in the organization of information in order to gain a more clear mental picture of the control situation

The important implications of these data are in the efforts to design automated alternatives to the current FPS marking procedures. Design alternatives need to take into account the benefits provided by these types of marks. For instance, the communication benefits provided by markings will not be replicated if both R-side and D-side controllers don't have access to the information display. Similarly, the memory benefits of markings will not be replicated if updated information is not easily viewed. For instance, some information may only be available within an automated system during specific operating modes and may not be available and easily viewed at all times. Thus, the updated information may not provide the same prospective memory benefits as a distinctive strip marking.
While each of the markings and their associated benefits may not be accounted for in the design process of an automated information system, the current observational and interviews study should provide valuable information to designers in their efforts to prioritize design tradeoffs. Particularly, design efforts should seek to maximize the memory and communicative benefits of the automated system.

\section{REFERENCES}

Albright, C. A., Truitt, T. R., Barile, A. L., Vortac, O. U., \& Manning, C. A. (1995) Controlling traffic without flight progress strips: Compensation, workload, performance, and opinion. Air Traffic Control Quarterly, 2, 229-248.

Geiselman, R. E. \& Fisher, R. P. (1997). Ten years of cognitive interviewing. In D. Payne \& F. Conrad (Eds.) Intersections in Basic and Applied Memory Research (pp. 291-310). Mahwah, NJ: Erlbaum.

Hopkin, V. D. (1995). Human factors in air-traffic control. London: Taylor \& Francis.

Hutchins, E. (1995). How a cockpit remembers its speeds. Cognitive Science, 19, 265-288.

Klein, G. A., Calderwood, R. \& MacGregor, D. (1989). Critical decision method for eliciting knowledge. IEEE Transactions on Systems, Man and Cybernetics Special Issue: Perspectives on Knowledge Engineering, 19(3), 462-472.

Luff, P., Heath, C., and Greatbatch, D. (1992). Tasks-in-Interaction: Paper and screen based documentation in collaborative activity. Conference Proceedings on Computer Supported Cooperative Work, 163-170.

Vortac, O. U., Barile, A. L., Albright, C., Truitt, T., Manning, C. A., \& Bain, D. (1996). Automation of flight data in air traffic control. In D. Herrmann, C. McEvoy, C. Hertzog, P. Hertel, \& M. K. Johnson (Eds.), Basic and Applied Memory Research, Vol. 2.,(pp. 353-366). Mahwah, NJ: Erlbaum.

Vortac, O. U., Edwards, M. B., Fuller, D. K., and Manning, C. A. (1993). Automation and cognition in air traffic control: An empirical investigation. Applied Cognitive Psychology Special Issue: Practical aspects of memory: The 1994 conference and beyond, 7, 631-651. 